\title{
Success Rates in Horizon 2020
}

\author{
Dr. Seán McCarthy \\ Hyperion Ltd, Watergrasshill, Co-Cork, Ireland \\ sean .mccarthy@ hyperion. ie \\ Letter from Industry
}

\begin{abstract}
Horizon 2020 is the name of the European Union's research and innovation programme. In the first three years of the programme (2014-2016) over 100,000 proposals were received. 10,456 proposals were selected for funding. This success rate $(11 \%)$ sounds very low and can discourage researchers from applying to the programme. This article argues that this success rate is not a reliable indicator for researchers. This article will show that a more realistic success rate is $29 \%$. In one sub-programme (Energy) a success rate of $47 \%$ was measured. The message from this paper is that the chances of success in Horizon 2020 depends on two main criteria: a) having an excellent scientific idea; and b) having a thorough understanding of the evaluation process.
\end{abstract}

Keywords. Horizon 2020, Success Rate, Evaluation Process.

\section{Introduction}

Horizon 2020 has a budget of $€ 77$ billion and this will be allocated to successful proposals approved in the period 2014 to 2020. The programme is divided into many sub-programmes. The most famous of these is the European Research Council (ERC) that supports fundamental research. Another well known programme is the Marie Curie Actions that supports PhD and Post Doctoral training. Details of all the different programmes can be found on the Horizon 2020 official website. ( http://ec.europa.eu/programmes/horizon2020/h2020-sections ). Other sub-programmes include research into health, food, energy, transport, security, and social sciences and humanities.

Every year 'calls for proposals' are published for each of these individual subprogrammes. Researchers submit proposals (individually and as part of consortia). The proposals are evaluated by independent evaluators. Successful proposers are invited to prepare legal agreement with the European Commission.

In 2017 a 'mid-term evaluation' of Horizon 2020 was undertaken to report on the progress of Horizon 2020 in the first three years (2014-2016). Arguments presented in this paper are also based on data used from this official report.

Every year the European Commission published a report on the progress of the programme entitled the 'Annual Monitoring Report'. Data used in this paper is from the most recent Annual Monitoring Report (2015). 


\section{The Evaluation Process in Horizon 2020}

Before discussing success rates it is important to understand the evaluation processes used in Horizon 2020. The evaluation process varies between the different programmes. In this paper a general evaluation process is described. When a proposal arrives in Brussels it is first checked by a Commission official against 'eligibility' criteria. 'Eligible' means that the forms were filled in properly. A proposal based on a very weak scientific idea can be classified as 'eligible' if the forms are filled in properly and the basic rules are met.

When the European Commission quotes 'success rates' they are based on these 'eligible proposals'. Clearly this is not a good denominator.

The 'eligible' proposals are then sent to independent scientific evaluators. The evaluators read the proposals individually. In some progammes the evaluators meet (Consensus meeting) and in other cases they simply send their evaluation scores to the relevant administrative body in Brussels. A final score is agreed based on the evaluators individual scores. A 'threshold' is set to define 'low quality proposals'. For example, in some programmes the maximum score is 15 and a threshold is set at 10 . Any proposal scoring below 10 is considered low quality and automatically elimated. Scoring below 10 is a very low score. These proposals should be classified as 'low quality proposals'. If research organisations had effective quality control procedures, these proposals would never have been submitted.

In the case of the ERC Programme the maximum score is 8 and a threshold of 4 is set. This threshold is sub-divided i.e. a threshold of $2 / 4$ for the researcher and a threshold of $2 / 4$ for the idea.

Following this evaluation a list of 'high quality proposals' is compiled. The final selection of successful proposals is based on these high quality proposals. This is a far better denominator to use when calculating the success rates

\section{Success Rates in Horizon 2020 (2014-2016)}

Total number of proposals received (2014-2016) $\quad=>104,000$

Total number of 'eligible proposals'

$=102,076$

(This means that over 2000 proposals did not fill in the forms properly)

Proposals below threshold (low quality proposals) $\quad=56,444$

(55.3\% of eligible proposals)

High Quality Proposals (above threshold)

$=45,632$

Proposals selected for funding

$=11,108$

Reported Success rate (funded/eligible)

$=10.88 \%(11,108 / 102,076)$

Real Success rate(funded/high quality proposals)

$=24.34 \%(11,108 / 45,632)$

This overall success rate of $24.34 \%$ is a far more encouraging number for researchers thinking about submitting proposals. 


\section{ERC Starting Grants (2014-2016)}

Source: European Commission (EC) Mid-Term Review of Horizon 2020 (Table 8 and 9)

The ERC Starting grant is one of the most prestigious grants for the career development of young researchers. Proposals are submitted by individual researchers.

The maximum score that reviewers can award ERC proposals is 8 ( 4 for the researcher, 4 for the research idea). Proposals below the threshold (with a score $<4$ ) in ERC are given a Grade $\mathrm{C}$. Any researcher receiving a grade $\mathrm{C}$ is not allowed to resubmit a proposal to ERC for two years.

Total number of ERC Starting grant proposals received (2014-20160)

Proposals below the threshold (Grade C)

$$
=6120
$$

High Quality Proposals (above threshold)

(68.4\% of eligible proposals)

Proposals funded

$$
\begin{aligned}
& =2827 \\
& =950 \\
& =10.6 \%(950 / 8947) \\
& =33.6 \%(950 / 2827)
\end{aligned}
$$

Reported Success rate (funded/eligible)

Real Success rate(funded/high quality proposals)

chers planning a career This success
in science.

\section{$4 \quad$ Success Rates Horizon 2020 (2015)}

The Annual Monitoring Report (2015) provides far more detailed data on individual programmes. It is the most recent Annual Monitoring Report available at the time of writing this article.

Total number of proposals received in 2015

Proposals below threshold (low quality proposals)

High Quality Proposals (above threshold)

Proposals selected for funding

Reported Success rate (funded/eligible)

Real Success rate(funded/high quality proposals)

ERC Statistics for 2015

Total number of ERC proposals received in 2015

Proposals below the threshold (Grade C)

High Quality Proposals (above threshold)

Proposals funded

Reported Success rate (funded/eligible)

Real Success rate(funded/high quality proposals)
$=42,535$

$=22,511$ ( $53 \%$ of eligible proposals)

$=20,024$

$=4,565$

$=10.7 \%(4565 / 42535)$

$=22.8 \%(4565 / 20024)$

$=10,019$
$=6083(61 \%$ of eligible proposals!! $)$
$=3936$
$=1327$
$=13.3 \%(1327 / 10019)$
$=33.7 \%(1327 / 3936)$




\section{SME Instrument (2015) (Page 122)}

An SME Instrument is a special grant for Small and Medium Sized Enterprises (SME). Companies submit proposals individually (no mandatory partners required).

Total number of proposals received in 2015

$$
\begin{aligned}
& =11008 \\
& =8378 \text { ( } 76 \% \text { of eligible proposals) }
\end{aligned}
$$

Proposals below the threshold

(Here the threshold is $<13 / 15$ for Phase I grants)

High Quality Proposals (above threshold)

$$
\begin{aligned}
& =2630 \\
& =714 \\
& =6.5 \%(714 / 11008) \\
& =28.2 \%(714 / 2630)
\end{aligned}
$$

Reported Success rate (funded/eligible)

For companies considering a proposal for an SME instrument a thorough understanding of the evaluation process and the evaluation criteria is essential.

Table 1. Summary of Real Success Rates in the individual programmes in 2015 (SME Instrument Proposals are excluded from the different programmes)

\begin{tabular}{lccccc}
\hline Programme & $\begin{array}{c}\text { Number of } \\
\text { Proposals }\end{array}$ & $\begin{array}{c}\text { Below } \\
\text { Threshold }\end{array}$ & $\begin{array}{c}\text { High Quality } \\
\text { Proposals }\end{array}$ & $\begin{array}{c}\text { Funded } \\
\text { Proposals }\end{array}$ & $\begin{array}{c}\text { Real Success } \\
\text { Rates }\end{array}$ \\
\hline Health (page 132) & 1212 & $75 \%$ & 318 & 94 & $29.5 \%$ \\
\hline Energy (page 145) & 839 & $71 \%$ & 243 & 114 & $47 \%$ \\
\hline Food ( page 138) & 358 & $36 \%$ & 228 & 62 & $27 \%$ \\
\hline Transport (page 153) & 702 & $33 \%$ & 467 & 167 & $35 \%$ \\
\hline Climate (page 160) & 648 & $48 \%$ & 335 & 79 & $23.5 \%$ \\
\hline Security (page 172) & 463 & $45 \%$ & 252 & 39 & $15.4 \%$ \\
\hline
\end{tabular}

\section{Conclusions}

\subsection{Message for the European Commission}

Why does the European Commission continue to quote success rates based on 'eligible' proposals? This number is meaningless. Success rates should be calculated as a percentage of 'High Quality Proposals' - those that score above the threshold. This is how 2015 results should be reported:

"In 2015 a total of 42,535 proposals were submitted to Horizon 2020. After evaluation by independent experts, $22,511(53 \%)$ were classified as 'low quality proposals'. From the remaining 'High Quality Proposals' $(20,024)$ a total of 4,565 proposals were accepted for funding. This represents a $22.8 \%$ success rate."

\subsection{Message for Research Organisations}

Research Organisations must take a share of the responsibility for the large numbers of low quality proposals submitted. Quality control procedures such as screening and 
proposal clinics, should identify weak proposals - before any considerable effort can be wasted on their preparation. This should be a core activity of Research Support Offices.

\subsection{Messages for Researchers}

Researchers must bear in mind the words of the Greek statesman and orator Pericles (450BC) "Having knowledge but lacking the power to express it clearly is no better than never having any ideas at all."

In a lecture you tell or express the idea. In a competitive proposal you have to sell the idea to the evaluators.

It is essential to understand how different types of evaluators think and how decisions are made in the evaluation process. There are two ways to learn this - attend training courses or (better) become an evaluator.

\section{$6 \quad$ References}

Official Website of Horizon 2020. Access 10th January2018: http://ec.europa.eu/programmes/horizon2020/

Projects funded in Horizon 2020. Accessed 10th January2018: http://cordis.europa.eu/projects/home_en.html

Mid-Term Review of Horizon 2020. Accessed 10th January2018: https://ec.europa.eu/research/evaluations/pdf/book_interim_evaluation_horizon_ 2020.pdf

Annual Monitoring Report of Horizon 2020 (2015) Accessed 10th January2018: https://ec.europa.eu/programmes/horizon2020/en/news/horizon-2020monitoring-report-2015 